\title{
Modern dietary pattern is prospectively associated with earlier age at menarche: data from the CHNS 1997-2015
}

\author{
Ruonan Duan', Yue Chen', Tian Qiao', Ruotong Duan², Mengxue Chen', Li Zhao', Yunhui Gong ${ }^{3,4}$ and \\ Guo Cheng ${ }^{5^{*}}$
}

\begin{abstract}
Background: Early age at menarche is associated with risk of several chronic diseases. Prospective study on the association between dietary pattern and timing of menarche is sparse. We examined whether dietary patterns prior to the menarche onset were prospectively associated with menarcheal age in Chinese girls.

Methods: One thousand one hundred eighteen girls aged 6-13 y in the China Health and Nutrition Survey (CHNS) with three-day 24-h recalls and information on potential confounders at baseline were included in the study. Dietary patterns were identified using principal component analysis. Age at menarche was self-reported at each survey. Cox proportional hazard regression models were performed to examine the associations of premenarcheal dietary patterns and menarcheal timing. Hazard ratios (HRs) and 95\% confidence intervals (Cls) were calculated.

Results: Three major dietary patterns were identified: modern dietary pattern, animal food pattern, and snack food pattern. After adjustment for age at baseline, region, ethnicity, maternal education level, energy intake at baseline, and body mass index Z-score at baseline, girls in the highest quartile of modern dietary pattern score had a 33\% higher probability of experiencing menarche at an earlier age than those in the lowest quartile (HR: 1.33, 95\% Cl: 1.002-1.77, $p$ for trend $=0.03$ ). No significant association was found for the animal food pattern or snack food pattern.
\end{abstract}

Conclusions: Higher adherence to modern dietary pattern during childhood is associated with an earlier menarcheal age. This association was independent of premenarcheal body size.

Keywords: Dietary patterns, Menarche, Cohort study, China health and nutrition survey

\section{Background}

Early age at menarche is a risk factor for insulin resistance [1], type 2 diabetes [2, 3], cardiovascular diseases [4] and hormone-related cancers [5-7]. Data from previous studies have consistently pointed to the fact that there appears to be a trend towards earlier attainment of

\footnotetext{
*Correspondence: ehw_cheng@126.com

${ }^{5}$ Laboratory of Molecular Translational Medicine, Center for Translational Medicine, Key Laboratory of Birth Defects and Related Diseases of Women and Children (Sichuan University), Ministry of Education, Department of Pediatrics, West China Second University Hospital, Sichuan University, Chengdu, Sichuan 610041, PR China

Full list of author information is available at the end of the article
}

menarche among Chinese girls $[8,9]$. Considering the potentially adverse consequences of early menarche for health in later life, identifying modifiable factors influencing the timing of menarche is of major public health relevance.

Large numbers of observational studies have addressed the role of dietary factors for menarche onset. Girls with higher intakes of fat [10] and animal protein [11, 12] had earlier menarche, while those with higher intakes of isoflavone [13], dietary fiber [14, 15] and vegetable protein [12] experienced their menarche at a later age. However, most of the existing studies focused on a single or a few 
nutrients or food groups. It is conceivable that nutrients and/or foods may influence menarche onset through their combined effects. Dietary pattern analysis, which examines the effects of overall diet, may be more predictive of disease risks than individual nutrients or foods [16]. To date, only two prospective observational studies conducted in Western children $[17,18]$ have investigated the longitudinal effects of dietary patterns on pubertal development. However, as general genetic background, food supplies, dietary patterns, and age at menarche vary by country and population, generalizability of those existing findings to a population of Chinese girls is uncertain.

In recent decades, China has experienced a remarkable transition in the structure of food consumption, such as increased consumption of animal-source foods, food away from home, and declining consumption of coarse grains and legumes [19]. Knowledge on specific type of dietary patterns might influence age at menarche among Chinese girls is lacking. A recent cross-sectional study of children in Shanghai found that unhealthy diet pattern, characterized by high intakes of dessert/snacks, soft drinks, and fried food, was positively associated with precocious puberty in girls [20]. However, this study was cross-sectional, and did not adjust for other potential confounding factors, such as energy intake. Using prospectively collected data from the China Health and $\mathrm{Nu}$ trition Survey (CHNS), we thus aimed to examine the prospective associations between dietary patterns during childhood and timing of menarche in Chinese girls.

\section{Methods}

\section{Study population}

We used data from the recent seven waves $(1997,2000$, 2004, 2006, 2009, 2011 and 2015) of the CHNS, an ongoing longitudinal cohort study which was started in 1989. Details on the study protocol have been described elsewhere [21]. In brief, a multistage, random cluster procedure was used to obtain nationally representative information on economy, socio-demography, nutrition, lifestyle and health issues in both urban and rural areas in 15 provinces and municipal cities in China. The CHNS is a community and household based study. In each community, 20 households were randomly selected, and all household members were invited to participate in the study. The study was approved by the Institutional Review Board at the University of North Carolina and the National Institute of Nutrition and Health, Chinese Center for Disease Control and Prevention. All parents provided written informed consent for their children's participation in the survey.

Between the 1997 and the 2015 survey, there were 2259 girls with plausible data on menarche. Of these, 1434 girls aged 6-13 y who had baseline dietary information and at least one follow-up visit of menarche onset thereafter were included. We excluded 9 girls who provided dietary records with extremely low or high total energy intake values $(<400 \mathrm{kcal} / \mathrm{d}$ or $>4000 \mathrm{kcal} / \mathrm{d})$ [13]. Three hundred seven participants were further excluded due to incomplete socio-demographic $(n=206)$ and anthropometric $(n=101)$ data. Finally, the present analysis was based on a sample of 1118 girls (Fig. 1).

\section{Dietary intake data}

Dietary intake data of girls in the CHNS were collected by trained investigators using three consecutive 24-h recalls. When girls were 12 years or older, they were asked to recall their consumption of all foods and beverages. For girls $<12$ y of age, their parents or guardians provided the information on food consumption at home, while girls provided the dietary intake information away from home. Food models and picture aids were used to improve the accuracy of the portion-size estimates [22]. The majority of the participants had a complete threeday $24-h$ recalls $(n=1098,98.2 \%$, i.e. $49.0 \%$ had three weekdays, $27.5 \%$ had two weekdays and one weekend day, and $21.7 \%$ had one weekday and two weekend days); the reminder had either one or two recalls $(n=4$ and $n=16$, respectively). Food intake was converted into energy and nutrient intake data using the Chinese Food Composition Tables (FCT) [23-26]. FCT 1991 was used in the 1997 and 2000 dietary survey; FCT 2002, FCT 2004 and 2009 (combined) were used in the 2004, 2006, 2009, and 2011 dietary survey.

\section{Dietary pattern derivation}

Dietary intake data were divided into 18 categories (Table 1) based on their similarity in nutrient profiles and Chinese Food Composition Tables. Principal components analysis (PCA) was conducted to identify dietary patterns at baseline through the PROC FACTOR procedure in SAS software (version 9.3, SAS Institute Inc., Cary, NC, USA.). Results of the Kaiser-Meyer-Olkin test $(0.65)$ and the Bartlett's test of sphericity $(p<0.0001)$ indicated that the present food intake data were suitable for factor analysis. Factors were rotated orthogonally to simplify the interpretation. Based on the eigenvalues (> 1 ), the inspection of scree plot, and the interpretability of the factors, three factors (dietary patterns) were retained. The factor loadings represent the correlations of each food group with the corresponding dietary pattern. Food groups with factor loadings $>0.30$ or $<-0.30$ were considered to be strongly associated within a pattern, and thus were selected to describe the dietary patterns. Labeling of the factors was primarily descriptive, and was based on our interpretation of the pattern structures [27]. Furthermore, the factor scores of each dietary pattern were calculated for each participant by 
2259 girls with data on menarcheal timing between 1997 and 2015 CHNS

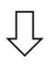

\section{Excluded:}

$n=825$ without dietary intake data at baseline $(6-13$ y)

$\stackrel{n}{n}=9$ had implausible energy intake in 24 -hour recalls

$\stackrel{\leftrightarrow}{n}=206$ with incomplete socio-demographic data

$\stackrel{n}{a}=101$ with incomplete anthropometric data

$\sqrt{2}$

1118 girls included in this analysis

Fig. 1 Flow chart for the study sample

summing the product of a standardized gram of each item consumed by its factor loading [28], with a higher factor score indicating a higher adherence to the corresponding dietary pattern.

\section{Assessment of age at menarche}

Girls aged 8 years or older and/or their parents were asked whether menarche had already occurred during each survey, and if they had, the month and year of their first menstrual period was recorded. If girls provided different menarcheal ages in different survey years, only the first reported menarcheal age in the panel data were used for analysis to reduce potential recall bias. For the present analysis, our outcome of interest was the time for the participants to experience menarche. Thus, for girls who experienced menarche during the follow-up survey, the observation time interval was from baseline to age of first menstrual period. For girls who did not reach menarche during the follow-ups, they were censored at the last follow-up visit, i.e. the observation time was from baseline to the last follow-up visit date.

Table 1 Food or food groups used in the dietary pattern analysis

\begin{tabular}{|c|c|}
\hline Food or food groups & Food items \\
\hline Cereals & Rice, noodle, steamed bun, corn, barley, millet, brown rice, black rice \\
\hline Tubers and starches & Potato, sweet potato, cassava, konjac powder, vermicelli \\
\hline Legumes and its products & Dried legumes, tofu, soya-bean milk, dried bean curd, mung bean, red bean \\
\hline Vegetables & $\begin{array}{l}\text { Root vegetable, leguminous vegetable and sprout, cucurbitaceous and solanaceous } \\
\text { vegetable, steam, leafy and flowering vegetable, aquatic vegetable }\end{array}$ \\
\hline Fungi and algae & Mushroom, agaric, tremella, laver, sea-tangle \\
\hline Fruits & Kernel fruit, drupe fruit, berry, orange fruit, tropic fruit, melons \\
\hline Nuts & Walnut, melon seeds, cashew, hazelnut, almond, pistachio \\
\hline Meat and its products & Pork, beef, mutton, rabbit meat, processed pork, sausage \\
\hline Poultry and its products & Chicken, duck, goose, turkey, pigeon \\
\hline Dairy products & Milk, dried milk, yoghurt, cheese \\
\hline Eggs & Chicken egg, duck egg, goose egg, partridge egg \\
\hline Fish and shellfish & Fish, shrimp, crab, shellfish \\
\hline Ethnic foods and cakes & Pancake, tangyuan, spring rolls, mooncake, tea-oil tree, mung bean cake \\
\hline Fast foods & Hamburger, sandwich, hotdog, chips, instant noodles, bread, biscuit, snacks \\
\hline Beverages & $\begin{array}{l}\text { Carbonated drink, fruit juice, vegetable juice, milk drink, vegetable protein drink, tea drink, } \\
\text { powdered drink, popsicle and ice cream }\end{array}$ \\
\hline Sugar and preserves & Lollipops, hard candy, chocolate, filled candy, honey, preserved fruit \\
\hline Fats and oils & Animal fat, vegetable oils \\
\hline Condiments & Sauce, vinegar, catsup, fermented soybean curd, pickles, spice, salt \\
\hline
\end{tabular}




\section{Covariates}

Detailed information on participants' socio-demographic characteristics was collected using a structured questionnaire at baseline, including birth year, ethnicity (Han and minority), residency (urban and rural), region (northeastern area: Liaoning, Heilongjiang; east coast area: Beijing, Jiangsu, Shandong, Shanghai; central area: Henan, Hubei, Hunan; and western area: Chongqing, Guangxi, Guizhou), household income (continuous variable), and maternal education level (illiterate, primary school, middle school, high school, technical or vocational degree, and college degree or higher).

Anthropometric measurements of the participants were performed at each visit by trained research assistants according to standard procedures, with the girls dressed in underwear only and barefoot. Height and weight were measured to the nearest $0.1 \mathrm{~cm}$ and $0.1 \mathrm{~kg}$, respectively. Body Mass Index (BMI) was calculated as weight divided by the square of height $\left(\mathrm{kg} / \mathrm{m}^{2}\right)$. Agespecific BMI Z-score were calculated for each participant using the equation by Cole et al. [29] based on a Chinese reference population [30].

\section{Statistical analysis}

All statistical analyses were performed with SAS procedures (version 9.3, 2011, SAS Institute Inc., Cary, NC, USA.). Results were considered statistically significant when a two-sided $p$-value $<0.05$.

We performed time-to-event analysis to investigate the prospective relevance of dietary pattern scores at baseline with the event of menarche using Cox proportional hazard regression models (PROC PHREG procedure in SAS software), which appropriately account for both the information on age at menarche from postmenarcheal girls and the censoring information from premenarcheal girls. The independent variables in the Cox proportional hazard regression models were the quartiles of each dietary pattern factor score. Hazard ratios (HRs) and 95\% confidence intervals (CIs) were calculated by comparing the second, third and fourth quartiles to the first quartile (as the reference category) of each dietary pattern factor score. Also, the associations between the three dietary pattern scores on a continuous scale and menarche onset were examined. Three models were used in our study: model 1 adjusted for age at baseline, region, ethnicity and maternal education level; model 2 further adjusted for energy intake at baseline. As we were interested in the potential mediating effect of body size on diet-menarche relations, we further adjusted for BMI Zscore at baseline in model 3.

\section{Sensitivity analysis}

Considering household income is a most frequently used proxy of socioeconomic status in investigating diet- menarche relations, we conducted sensitivity analysis with substitution of household income per capita (continuous variable) for maternal educational level in order to obtain more comparable results.

\section{Results}

\section{Characteristics of study participants}

General characteristics of the study sample are shown in Table 2. Girls included in the present analyses $(n=1118)$ were $8.3 \pm 1.8$ years old at baseline. Among them, 711 participants $(63.6 \%)$ reported menarche during followup, and 407 participants (36.4\%) were censored at the time of last follow-up visit. Overall, the participants were followed up for $4.0 \pm 1.8$ years after study baseline. The mean length of follow-up was longer for girls who had reached menarche $(4.1 \pm 1.8$ y) during the follow-up than their counterparts who were censored $(3.8 \pm 1.7 \mathrm{y})$. The mean menarcheal age from 711 postmenarcheal girls was $12.7 \pm 1.2$ years. Age at menarche did not differ between the 711 girls and the 656 postmenarcheal girls who also had data on menarcheal age but were excluded from the final analysis due to lack of dietary intake at baseline, socio-demographic and anthropometric data $(p=0.53)$.

\section{Dietary patterns based on principal component analysis}

The factor loadings for the three main dietary patterns are shown in Table 3. Factor 1 (the modern dietary pattern) was characterized by high intakes of fast foods, dairy products, fruits and eggs, and low intakes of cereals, vegetables, and condiments. Factor 2 (the animal food pattern) was loaded heavily for meat, poultry, fish and shellfish. Factor 3 (the snack food pattern) was marked by high intakes of nuts, beverages, ethnic foods and cakes, and legumes. These three dietary patterns explained $27.2 \%$ of the total variation in dietary intake (13.1, 7.5 and $6.6 \%$ for factor 1 , factor 2 and factor 3 , respectively).

\section{Prospective associations between three dietary patterns and menarche}

Cox proportional hazard regression models for the associations between the three dietary pattern scores at baseline and age at menarche are presented in Table 4. There was a positive association between the modern dietary pattern score and probability of earlier menarche, which remained significant when the potential mediator BMI Z-score at baseline was included in the final model (adjusted HR in model 3: 1.13, 95\% CI: 1.03-1.24). After adjustment for age at baseline, region, ethnicity, maternal education level, energy intake at baseline, and BMI Z-score at baseline (model 3), girls in the highest quartile of modern dietary pattern score had a 33\% higher probability of experiencing menarche at an earlier age 
Table 2 General characteristics of the CHNS participants in the present study

\begin{tabular}{|c|c|}
\hline Characteristics & Values $($ mean $\pm \mathrm{SD} / \mathrm{n}(\%))$ \\
\hline $\mathrm{n}$ & 1118 \\
\hline postmenarcheal girls during follow-up, $n$ & $711(63.6)$ \\
\hline Age at baseline ${ }^{a}, y$ & $8.3 \pm 1.8$ \\
\hline Years of follow-up, y & $4.0 \pm 1.8$ \\
\hline \multicolumn{2}{|l|}{ Wave (baseline survey year) } \\
\hline 1997 & $488(43.6)$ \\
\hline 2000 & $170(15.2)$ \\
\hline 2004 & $177(15.8)$ \\
\hline 2006 & $66(5.9)$ \\
\hline 2009 & $100(8.9)$ \\
\hline 2011 & $117(10.5)$ \\
\hline \multicolumn{2}{|l|}{ Region ${ }^{b}$} \\
\hline Northeastern area & $199(17.8)$ \\
\hline East coast & $210(18.8)$ \\
\hline Central area & $359(32.1)$ \\
\hline Western area & $350(31.3)$ \\
\hline \multicolumn{2}{|l|}{ Residency } \\
\hline Urban & $343(30.7)$ \\
\hline Rural & $775(69.3)$ \\
\hline \multicolumn{2}{|l|}{ Ethnicity } \\
\hline Han & $950(85.0)$ \\
\hline Minority & $168(15.0)$ \\
\hline \multicolumn{2}{|l|}{ Maternal education level } \\
\hline Illiterate & $194(17.4)$ \\
\hline Primary school & $283(25.3)$ \\
\hline Middle school & $404(36.1)$ \\
\hline High school & $140(12.5)$ \\
\hline Technical or vocational degree & $45(4.0)$ \\
\hline College degree or higher & $52(4.7)$ \\
\hline Energy intake at baseline, $\mathrm{kcal} / \mathrm{d}$ & $1546 \pm 495$ \\
\hline Protein, \% of energy & $12.4 \pm 3.0$ \\
\hline Fat, \% of energy & $28.1 \pm 11.8$ \\
\hline Carbohydrate, \% of energy & $59.5 \pm 12.3$ \\
\hline Weight, kg & $25.9 \pm 7.3$ \\
\hline Height, cm & $127.2 \pm 12.4$ \\
\hline BMI Z-score at baseline ${ }^{c}$ & $-0.04 \pm 1.22$ \\
\hline
\end{tabular}

${ }^{a}$ Mean age at recruitment

${ }^{b}$ Twelve provinces were categorized into four regions: central area (Henan, Hubei, Hunan), east coast (Beijing, Jiangsu, Shandong, Shanghai), northeastern area (Liaoning, Heilongjiang), and western area (Chongqing, Guangxi, Guizhou) ' BMI Z-score was calculated using the equation by Cole et al. [29] based on a Chinese reference population [30]

than those in the lowest quartile (adjusted HR: 1.33, 95\% CI: $1.002-1.77, p$ for trend $=0.03$ ). However, no significant association was observed for animal food pattern or
Table 3 Orthogonally rotated factor loadings for three dietary patterns derived from principal components analysis ${ }^{\text {a }}$

\begin{tabular}{llll}
\hline Food or food groups & $\begin{array}{l}\text { Factor 1: } \\
\text { Modern }\end{array}$ & $\begin{array}{l}\text { Factor 2: } \\
\text { Animal food }\end{array}$ & $\begin{array}{l}\text { Factor 3: } \\
\text { Snack food }\end{array}$ \\
\hline Cereals & $-\mathbf{0 . 6 3}$ & 0.10 & -0.12 \\
Tubers and starches & 0.06 & -0.21 & 0.00 \\
Legumes & -0.11 & -0.06 & $\mathbf{0 . 4 0}$ \\
Vegetables & $-\mathbf{0 . 5 3}$ & 0.21 & -0.13 \\
Fungi and algae & 0.14 & 0.30 & -0.04 \\
Fruits & $\mathbf{0 . 3 1}$ & 0.21 & 0.11 \\
Nuts & -0.11 & 0.10 & $\mathbf{0 . 6 8}$ \\
Meat and its products & 0.10 & $\mathbf{0 . 5 3}$ & 0.05 \\
Poultry and its products & 0.21 & $\mathbf{0 . 5 7}$ & -0.08 \\
Dairy products & $\mathbf{0 . 5 3}$ & 0.25 & 0.19 \\
Eggs & $\mathbf{0 . 3 1}$ & 0.25 & 0.22 \\
Fish and shellfish & -0.02 & $\mathbf{0 . 5 3}$ & 0.17 \\
Ethnic foods and cakes & 0.25 & -0.03 & $\mathbf{0 . 4 5}$ \\
Fast foods & $\mathbf{0 . 6 3}$ & 0.20 & -0.11 \\
Beverages & 0.15 & 0.03 & $\mathbf{0 . 4 8}$ \\
Sugar and preserves & 0.24 & 0.11 & -0.07 \\
Fats and oils & 0.01 & -0.20 & 0.15 \\
Condiments & $\mathbf{- 0 . 3 8}$ & 0.22 & 0.17 \\
\% Variance explained & $13.1 \%$ & $7.5 \%$ & $6.6 \%$ \\
\hline a The bold font was used for factor loadings & 0.30 or <-0.30 &
\end{tabular}

snack food pattern with timing of menarche. In sensitivity analyses, replacing maternal education level with household income per capita did not change these results (data not shown).

\section{Discussion}

In the present study, we identified three major dietary patterns in the years preceding onset of menarche among Chinese girls: modern dietary pattern, animal food pattern and snack food pattern. We found that higher adherence to modern dietary pattern was associated with higher odds of experiencing menarche at an earlier age. This association was independent of potential sociodemographic confounders and premenarcheal body size. However, no significant association was found for animal food pattern or snack food pattern.

To our knowledge, our study is the first prospective study of the associations between dietary patterns and timing of menarche in a Chinese population. Compared with studies focusing on single nutrients or foods, dietary pattern takes the interactions of nutrients or foods into account, and thus could have important public health implications because overall patterns of dietary intake might be easier for the public to translate into daily diets. It serves as a complementary approach to traditional analysis, and evidence could be enhanced 
Table 4 Cox proportional hazard regression models of three dietary patterns and timing of menarche among 1118 girls in the CHNS $^{\text {a }}$

\begin{tabular}{|c|c|c|c|}
\hline & Model $1^{b}$ & Model $2^{c}$ & Model $3^{d}$ \\
\hline \multicolumn{4}{|l|}{ Modern dietary pattern } \\
\hline Quartile 1 & 1.00 & 1.00 & 1.00 \\
\hline Quartile 2 & $0.98(0.79,1.21)$ & $1.04(0.83,1.30)$ & $1.03(0.82,1.28)$ \\
\hline Quartile 3 & $1.19(0.95,1.50)$ & $1.27(0.98,1.65)$ & $1.24(0.96,1.60)$ \\
\hline Quartile 4 & $1.23(0.95,1.59)$ & $1.34(1.01,1.78)$ & $1.33(1.002,1.77)$ \\
\hline Dietary pattern score (continuous) & $1.13(1.03,1.23)$ & $1.14(1.04,1.25)$ & $1.13(1.03,1.24)$ \\
\hline$p$ for trend ${ }^{e}$ & 0.03 & 0.02 & 0.03 \\
\hline \multicolumn{4}{|l|}{ Animal food pattern } \\
\hline Quartile 1 & 1.00 & 1.00 & 1.00 \\
\hline Quartile 2 & $1.05(0.84,1.30)$ & $1.07(0.85,1.33)$ & $1.06(0.85,1.32)$ \\
\hline Quartile 3 & $1.00(0.80,1.25)$ & $1.02(0.81,1.28)$ & $1.01(0.80,1.27)$ \\
\hline Quartile 4 & $1.12(0.89,1.41)$ & $1.14(0.88,1.47)$ & $1.12(0.87,1.45)$ \\
\hline Dietary pattern score (continuous) & $1.03(0.95,1.11)$ & $1.03(0.95,1.12)$ & $1.03(0.95,1.12)$ \\
\hline$p$ for trend ${ }^{\mathrm{e}}$ & 0.49 & 0.52 & 0.54 \\
\hline \multicolumn{4}{|l|}{ Snack food pattern } \\
\hline Quartile 1 & 1.00 & 1.00 & 1.00 \\
\hline Quartile 2 & $0.99(0.81,1.23)$ & $1.00(0.81,1.23)$ & $1.00(0.81,1.24)$ \\
\hline Quartile 3 & $0.91(0.73,1.13)$ & $0.91(0.73,1.13)$ & $0.91(0.74,1.14)$ \\
\hline Quartile 4 & $0.85(0.68,1.06)$ & $0.85(0.68,1.06)$ & $0.85(0.68,1.06)$ \\
\hline Dietary pattern score (continuous) & $0.93(0.85,1.02)$ & $0.93(0.85,1.02)$ & $0.93(0.85,1.01)$ \\
\hline$p$ for trend ${ }^{e}$ & 0.12 & 0.12 & 0.13 \\
\hline
\end{tabular}

${ }^{a}$ Values are hazard ratios (HRs) and $95 \%$ confidence intervals (95\% Cls)

${ }^{\mathrm{b}}$ Model 1 adjusted for age at baseline, region, ethnicity and maternal education level

c Model 2 adjusted for variables in model 1 and energy intake at baseline

${ }^{\mathrm{d}}$ Model 3 adjusted for variables in model 2 and BMI Z-score (continuous) at baseline

e $P$ for trends were tested with the quartiles of the three dietary pattern scores as continuous variables in the Cox proportional hazard regression models

when the results from multiple lines of research (i.e. nutrients, foods, and dietary patterns) are consistent [16].

The modern dietary pattern we identified showed some similarities with results previously reported by Zhang et al. [31], which was characterized by high intakes of fast foods, milk, fruits and eggs, and low intakes of grains and vegetables. We observed that modern dietary pattern was associated with an increased risk for accelerated menarche. This pattern was implicated in the timing of menarche probably in any of the three ways: high consumption of milk, high fat intake, and low consumption of plant foods. Previous studies have shown that consumption of milk contributed to high IGF-I concentrations in prepubertal children [32, 33], which was found to be associated with earlier menarche [34]. Also, high fat intake may be implicated in the earlier menarcheal timing due to its potential impact on estrogen metabolism [35]. In addition, intake of vegetable protein sources has been related to the delayed onset of puberty probably due to the high content of dietary fiber and isoflavones [35]. Taken together, these evidence suggest that modern dietary pattern has potential influence on menarcheal timing through metabolic changes in insulin-mediated pathway mechanisms and upregulation of hormones [20]. Since mid-1990s, a marked transition to the modern dietary pattern with increased consumption of sugary and fat-rich foods and declining consumption of coarse grains has occurred in China [22]. Ouyang el al [36]. reported that fruits, milk, and fast foods were the three most consumed snacks among Chinese children. Moreover, calories from fast foods account for $26-40 \%$ of total energy intake. Although more research is needed to determine the effects of modern dietary pattern on Chinese children's health, the present public health recommendations include the necessity to limit consumption of fast foods and to increase consumption of plant foods.

It is of note that the animal food pattern was not associated with age at menarche in the present study, although most of the previous studies have shown that girls with high intake of animal protein (especially red meat) reached menarche at an earlier age [37-40]. However, some evidence has indicated that animal protein intake or meat intake was not associated with timing of 
menarche [41, 42]. For example, using data from the Growing Up Today Study (GUTS), Carwile et al. [41] found that peripubertal total meat or red meat intake was not related to age at menarche in 5583 US girls aged 9-14 years, which was consistent with our finding. The discrepancies across different studies suggest that the effect of animal food groups on timing of menarche may be age-specific. In fact, some researchers have proposed that animal food intake at younger ages (e.g. early childhood) may be more relevant to occurrence of menarche than peripubertal intake [43]. However, the underlying mechanisms to illustrate these potential age-specific effects remain unknown. Another potential explanation is that the animal food pattern in our study was loaded heavily for both red meat and fish/shellfish, and these two types of meat may be associated with timing of menarche in opposite ways. For example, a prospective study conducted in 456 US girls aged 5-12 y showed that red meat was inversely related to age at menarche while tuna/sardine intake was positively associated with age at menarche [40]. Similarly, we speculate that the non-significant association for snack food pattern could potentially be driven by the counteraction of positive impact of nuts and legumes which are rich in vegetable protein, dietary fiber and isoflavones [12-14, 44], and negative impact of sugary-rich foods (i.e. beverages, ethnic foods and cakes) on menarcheal timing $[45,46]$. This may hence reflect a combined effect of different types of foods on menarche. However, the underlying mechanisms of the impact of snack food pattern on menarcheal timing require further study.

Our study has several strengths, including the prospective design and the representative sample from four different regions in China. Dietary intake data were collected by using a validated three-day 24-h dietary recalls. A further advantage lies in the use of dietary pattern analysis, which examines the effects of diet as a whole, and might be much easier for the public to interpret or translate into diets. In addition, the comprehensive and detailed data allowed us to simultaneously take a number of potential confounders or mediators into account and thus to reliably examine the association between dietary patterns and menarcheal timing.

Some limitations should be mentioned. First, menarche represents a relatively late stage of pubertal development. Although using menarcheal age as an indicator of puberty timing is reliable, the effects of dietary patterns on earlier events of pubertal development may differ from those on age at menarche. Future work should address the relevance of dietary patterns and early stage of puberty, such as the age at take-off (ATO, i.e. the age at minimal height velocity) [47]. Second, although the potential mediating effect of body size on diet-menarche relations was examined in our study, only BMI Z-score at baseline was considered instead of continuous change of body composition during the entire pubertal period. Furthermore, as data on parental pubertal characteristics (e.g. maternal age at menarche) is not available in the CHNS, genetic influences on menarcheal timing could not be adjusted for.

\section{Conclusions}

Our data suggest that girls with higher adherence to modern dietary pattern experienced menarche at an earlier age. This association was independent of body mass. Our finding provides evidence to support the recommendation to have a balanced diet for prepubertal girls in China. Further research is needed to address the prospective effects of dietary patterns on earlier stage of pubertal development in Chinese girl population, and to determine the underlying biologic mechanisms.

\section{Abbreviations}

CHNS: China Health and Nutrition Survey; BMI: Body mass index; FCT: Food Composition Tables; PCA: Principal components analysis; HR: Hazard ratio; $\mathrm{Cl}$ : Confidence interval

\section{Acknowledgements \\ This research uses data from the China Health and Nutrition Survey (CHNS). We thank the National Institute for Nutrition and Food Safety, China Center for Disease Control and Prevention, Carolina Population Center, the University of North Carolina at Chapel Hill, the NIH and the Fogarty International Center, NIH, for providing financial support for the CHNS data collection and analysis of files from 1989 to 2015 and future surveys.}

\section{Authors' contributions}

G.C. contributed to the conception and design of the study. R.D. conducted data analysis and wrote the manuscript. Y. C., T. Q., R.D., M.C., L.Z. and Y.G. contributed to analysis and interpretation of the data. All authors have critically reviewed the manuscript for important intellectual content, and given approval of the final version for publication.

\section{Funding}

This study was supported by the National Natural Science Foundation of China (81673158), International Cooperation Project of Science and Technology Department of Sichuan Province (19GJHZ0171), and International Cooperation Project of Chengdu Science and Technology Bureau (2019-GH02-00058-HZ).

\section{Availability of data and materials}

The data supporting the findings of this study are available from CHNS (https://www.cpc.unc.edu/projects/china/data/datasets).

\section{Ethics approval and consent to participate}

The CHNS study was approved by the Institutional Review Board at the University of North Carolina and the National Institute of Nutrition and Health, Chinese Center for Disease Control and Prevention. All parents provided written informed consent for their children's participation in the survey.

\section{Consent for publication}

Not applicable.

\section{Competing interests}

The authors declare that they have no competing interests.

\section{Author details}

${ }^{1}$ West China School of Public Health and West China Fourth Hospital, Sichuan University, Chengdu, PR China. ${ }^{2}$ Department of Clinical Medicine, Changzhi Medical College, Changzhi, Shanxi, PR China. ${ }^{3}$ Department of 
Obstetrics, West China Second University Hospital, Sichuan University, Chengdu, PR China. ${ }^{4}$ Key Laboratory of Birth Defects and Related Diseases of Women and Children (Sichuan University), Ministry of Education, Chengdu, PR China. ${ }^{5}$ Laboratory of Molecular Translational Medicine, Center for Translational Medicine, Key Laboratory of Birth Defects and Related Diseases of Women and Children (Sichuan University), Ministry of Education, Department of Pediatrics, West China Second University Hospital, Sichuan University, Chengdu, Sichuan 610041, PR China.

Received: 24 April 2020 Accepted: 2 September 2020

\section{Published online: 09 September 2020}

\section{References}

1. Zhang Z, Hu X, Yang C, Chen X. Early age at menarche is associated with insulin resistance: a systemic review and meta-analysis. Postgrad Med. 2019; 131(2):144-50

2. Yang L, Li L, Peters SAE, Clarke R, Guo Y, Chen Y, et al. Age at menarche and incidence of diabetes: a prospective study of 300,000 women in China. Am J Epidemiol. 2018;187(2):190-8.

3. Janghorbani M, Mansourian M, Hosseini E. Systematic review and metaanalysis of age at menarche and risk of type 2 diabetes. Acta Diabetol. 2014; 51(4):519-28.

4. Lee JJ, Cook-Wiens G, Johnson BD, Braunstein GD, Berga SL, Stanczyk FZ, et al. Age at menarche and risk of cardiovascular disease outcomes: findings from the National Heart Lung and blood institute-sponsored women's ischemia syndrome evaluation. J Am Heart Assoc. 2019:8(12):e012406.

5. Collaborative Group on Hormonal Factors in Breast C. Menarche, menopause, and breast cancer risk: individual participant meta-analysis, including 118964 women with breast cancer from 117 epidemiological studies. Lancet Oncol. 2012;13(11):1141-51.

6. Gong T-T, Wang Y-L, Ma X-X. Age at menarche and endometrial cancer risk: a dose-response meta-analysis of prospective studies. Sci Rep. 2015; 5:14051

7. Gong T-T, Wu Q-J, Vogtmann E, Lin B, Wang Y-L. Age at menarche and risk of ovarian cancer: a meta-analysis of epidemiological studies. Int J Cancer. 2013;132(12):2894-900.

8. Meng X, Li S, Duan W, Sun Y, Jia C. Secular trend of age at menarche in Chinese adolescents born from 1973 to 2004. Pediatrics. 2017;140(2): e20170085.

9. Song Y, Ma J, Wang H-J, Wang Z, Hu P, Zhang B, et al. Trends of age at menarche and association with body mass index in Chinese school-aged girls, 1985-2010. J Pediatr. 2014;165(6):1172-7.e1.

10. Merzenich $\mathrm{H}$, Boeing $\mathrm{H}$, Wahrendorf J. Dietary fat and sports activity as determinants for age at menarche. Am J Epidemiol. 1993;138(4):217-24.

11. Berkey CS, Gardner JD, Frazier AL, Colditz GA. Relation of childhood diet and body size to menarche and adolescent growth in girls. Am J Epidemiol. 2000;152(5):446-52.

12. Günther ALB, Karaolis-Danckert N, Kroke A, Remer T, Buyken AE. Dietary protein intake throughout childhood is associated with the timing of puberty. J Nutr. 2010;140(3):565-71.

13. Mervish NA, Teitelbaum SL, Pajak A, Windham GC, Pinney SM, Kushi LH, et al. Peripubertal dietary flavonol and lignan intake and age at menarche in a longitudinal cohort of girls. Pediatr Res. 2017;82(2):201-8.

14. Koo MM, Rohan TE, Jain M, McLaughlin JR, Corey PN. A cohort study of dietary fibre intake and menarche. Public Health Nutr. 2002;5(2):353-60.

15. Tian G, Liu Y, Xue HM, Luo J, Chen YR, Bao YX, et al. Dietary Fiber and pubertal development among children and adolescents--a cross-sectional study in Chengdu, Sichuan. Sichuan Da Xue Xue Bao Yi Xue Ban. 2016;47(2): 244-7.

16. Hu FB. Dietary pattern analysis: a new direction in nutritional epidemiology. Curr Opin Lipidol. 2002;13(1):3-9.

17. Szamreta EA, Qin B, Rivera-Núñez Z, Parekh N, Barrett ES, Ferrante J, et al. Greater adherence to a Mediterranean-like diet is associated with later breast development and menarche in peripubertal girls. Public Health Nutr. 2020;23(6):1020-30.

18. Jansen EC, Zhou L, Perng W, Song PX, Rojo MMT, Mercado A, et al. Vegetables and lean proteins-based and processed meats and refined grains -based dietary patterns in early childhood are associated with pubertal timing in a sex-specific manner: a prospective study of children from Mexico City. Nutr Res. 2018:56:41-50.
19. Popkin BM. Synthesis and implications: China's nutrition transition in the context of changes across other low- and middle-income countries. Obes Rev. 2014;Suppl 1(01):60-7.

20. Chen C, Chen Y, Zhang Y, Sun W, Jiang Y, Song Y, et al. Association between dietary patterns and precocious puberty in children: a populationbased study. Int J Endocrinol. 2018;4528704:7. https://doi.org/10.1155/2018/ 4528704.

21. Popkin BM, Du S, Zhai F, Zhang B. Cohort profile: the China health and nutrition survey--monitoring and understanding socio-economic and health change in China, 1989-2011. Int J Epidemiol. 2010;39(6):1435-40.

22. Zhai FY, Du SF, Wang ZH, Zhang JG, Du WW, Popkin BM. Dynamics of the Chinese diet and the role of urbanicity, 1991-2011. Obes Rev. 2014;Suppl 1(01):16-26.

23. Wang GY. China food composition. 1st ed. Peking: People's Medical Publishing House; 1991.

24. Yang YX, Wang GY, Pan XC. China food composition. 1st ed. Peking: Peking University Medical Press; 2002

25. Yang YX, He M, Pan XC. China food composition. 1st ed. Peking: Peking University Medical Press; 2004.

26. Yang YX, Wang GY, Pan XC. China food composition. 2nd ed. Peking: Peking University Medical Press; 2009.

27. Gong Y, Tian G, Xue H, Zhang X, Zhao Y, Cheng G. Higher adherence to the 'vegetable-rich' dietary pattern is related to longer telomere length in women. Clin Nutr. 2018;37(4):1232-7.

28. Bell LK, Schammer C, Devenish G. Dietary patterns and risk of obesity and early childhood caries in Australian toddlers: findings from an Australian cohort study. Nutrients. 2019;11(11):2828.

29. Cole TJ, Bellizzi MC, Flegal KM, Dietz WH. Establishing a standard definition for child overweight and obesity worldwide: international survey. BMJ. 2000; 320(7244):1240-3.

30. Li H, Ji C-Y, Zong X-N, Zhang Y-Q. Body mass index growth curves for Chinese children and adolescents aged 0 to 18 years. Zhonghua Er Ke Za Zhi. 2009;47(7):493-8.

31. Zhang J, Wang $H$, Wang $Y$, Xue $H$, Wang Z, Du W, et al. Dietary patterns and their associations with childhood obesity in China. Br J Nutr. 2015;113(12): 1978-84.

32. Qin LQ, He K, Xu JY. Milk consumption and circulating insulin-like growth factor-I level: a systematic literature review. Int J Food Sci Nutr. 2009; 60(Suppl 7):330-40.

33. Hoppe C, Mølgaard C, Juul A, Michaelsen KF. High intakes of skimmed milk, but not meat, increase serum IGF-I and IGFBP-3 in eight-year-old boys. Eur J Clin Nutr. 2004;58(9):1211-6.

34. Thankamony A, Ong KK, Ahmed ML, Ness AR, Holly JM, Dunger DB. Higher levels of IGF-I and adrenal androgens at age 8 years are associated with earlier age at menarche in girls. J Clin Endocrinol Metab. 2012;97(5):E786-90

35. Cheng G, Buyken AE, Shi L, Karaolis-Danckert N, Kroke A, Wudy SA, et al. Beyond overweight: nutrition as an important lifestyle factor influencing timing of puberty. Nutr Rev. 2012;70:133-52.

36. Ouyang Y, Wang H, Wang D, Wang Z, Zhang J, Du W, et al. Snacking food pattern among Chinese children and adolescents in twelve provinces. Wei Sheng Yan Jiu. 2016;45(6):868-75.

37. Rogers IS, Northstone K, Dunger DB, Cooper AR, Ness AR, Emmett PM. Diet throughout childhood and age at menarche in a contemporary cohort of British girls. Public Health Nutr. 2010;13(12):2052-63.

38. Luo J, Yang MZ, Duan RN, Tian G, Bao YX, Chen YR, et al. Consumptions of meat and dairy products, zinc intake and pubertal development in adolescents in Chengdu. Sichuan Da Xue Xue Bao Yi Xue Ban. 2015;46(5):726-31.

39. Yang $M$, Xue H, Duan R, Liu Y, Cheng G. Cross-sectional association between food rich in protein and pubertal development among children and adolescents in Chengdu City. Wei Sheng Yan Jiu. 2015;44(1):43-8.

40. Jansen EC, Marín C, Mora-Plazas M, Villamor E. Higher childhood red meat intake frequency is associated with earlier age at Menarche. J Nutr. 2015; 146(4):792-8.

41. Carwile JL, Willett WC, Wang M, Rich-Edwards J, Frazier AL, Michels KB. Milk consumption after age 9 years does not predict age at menarche. J Nutr. 2015;145(8):1900-8.

42. Khopkar S, Kulathinal S, Virtanen SM, Säävälä M. Age at menarche and diet among adolescents in slums of Nashik. India Int J Adolesc Med Health. 2015;27(4):451-6. 
43. Villamor E, Jansen EC. Nutritional determinants of the timing of puberty. Annu Rev Public Health. 2016;37:33-46.

44. Cheng G, Remer T, Prinz-Langenohl R, Blaszkewicz M, Degen GH, Buyken AE. Relation of isoflavones and fiber intake in childhood to the timing of puberty. Am J Clin Nutr. 2010;92:556-64.

45. Carwile JL, Willett WC, Spiegelman D, Hertzmark E, Rich-Edwards J, Frazier $\mathrm{AL}$, et al. Sugar-sweetened beverage consumption and age at menarche in a prospective study of US girls. Hum Reprod. 2015;30:675-83.

46. Mueller NT, Jacobs DR Jr, MacLehose RF, Demerath EW, Kelly SP, Dreyfus JG, et al. Consumption of caffeinated and artificially sweetened soft drinks is associated with risk of early menarche. Am J Clin Nutr. 2015;102:648-54.

47. Buyken AE, Karaolis-Danckert N, Remer T. Association of prepubertal body composition in healthy girls and boys with the timing of early and late pubertal markers. Am J Clin Nutr. 2009;89(1):221-30.

\section{Publisher's Note}

Springer Nature remains neutral with regard to jurisdictional claims in published maps and institutional affiliations.

Ready to submit your research? Choose BMC and benefit from:

- fast, convenient online submission

- thorough peer review by experienced researchers in your field

- rapid publication on acceptance

- support for research data, including large and complex data types

- gold Open Access which fosters wider collaboration and increased citations

- maximum visibility for your research: over $100 \mathrm{M}$ website views per year

At $\mathrm{BMC}$, research is always in progress.

Learn more biomedcentral.com/submissions 